\title{
Experimental and numerical analysis on PCB coupling in non uniform field environment
}

\author{
F. Sonnemann \\ Diehl Munitionssysteme GmbH \& Co. KG, Germany
}

\begin{abstract}
HPM and UWB pulses are capable of disrupting signal processing or even destroying semiconductor structures. Hence, it is useful to estimate the induced voltage levels on printed circuit boards (PCB) in response to different excitation signals. The considered PCB is integrated within a missile-like cylindrical structure, called GENEC, and is equipped with differential sensors to measure the $\mathrm{E}$ - and $\mathrm{H}$ field above the PCB. Standard transmission line theory is applied to develop a SPICE model that calculates the terminal voltages at the transmission line loads.
\end{abstract}

\section{Introduction}

Experimental HPM/UWB - coupling investigations have been shown that the circuit response strongly depends on the incident waveform, the overall system coupling behaviour and the demodulation capability of the electrical components themselves. In order to estimate and to model the demodulated circuit response, it is necessary to have knowledge about waveform and amplitude of the HF voltage signals induced in the printed circuit board (PCB) lines. This knowledge can also help to define an upper electrical fieldstrength value that mustn't be exceeded to avoid binary faults (e.g. logical faults reset faults etc.) and to avoid potential destruction effects of components. Furthermore, the knowledge about the amplitude of the induced voltage levels enable the possibility to define the degree of hardening measures and protection elements to keep the voltage level below a certain maximum threshold value in respect to the required EMC test standards. The following approach to estimate the induced voltage waveforms on the PCB combines PCB field measurement and a PCB transmission line model. The advantage of this procedure is a) the determination of the induced voltage

Correspondence to: F. Sonnemann

(frank.sonnemann@diehl-mun.de)

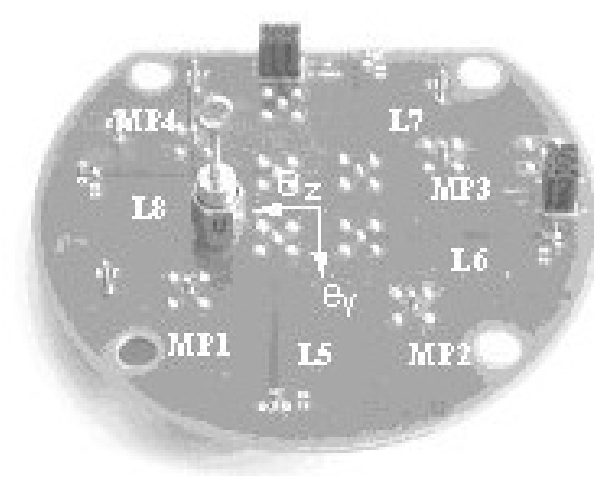

Fig. 1. Sensor board.

waveforms in PCB lines is non-interacting with the electrical circuit because the electric and magnetic field above the PCB will be measured and $b$ ) field measurements are in general easier to perform than high frequency voltage measurements.

Field coupling to transmission lines is treated in several papers (Paul, 1994; Leone and Singer, 1999; Erdin et al., 2001; Tesche, 1997) and the references therein and complete solutions are available for uniform plane excitation. For a rigorous analysis in a non-uniform field environment, field solvers have to be incorporated with conventional circuit simulators. The following proposed approach uses small (differential) sensors on the PCB to measure the electric and magnetic fields above the board in the time domain. An approximate SPICE coupling model is given, where the voltage probe signals can be directly embedded as voltage controlled voltage sources. Within the model, different types of loads can be treated to simulate e.g. the impedance characteristic of integrated circuits. For convenience, the analysis is restricted to a lossless, single line over a groundplane. Throughout this paper the transmission line formulation in terms of the "total voltage" is used. 

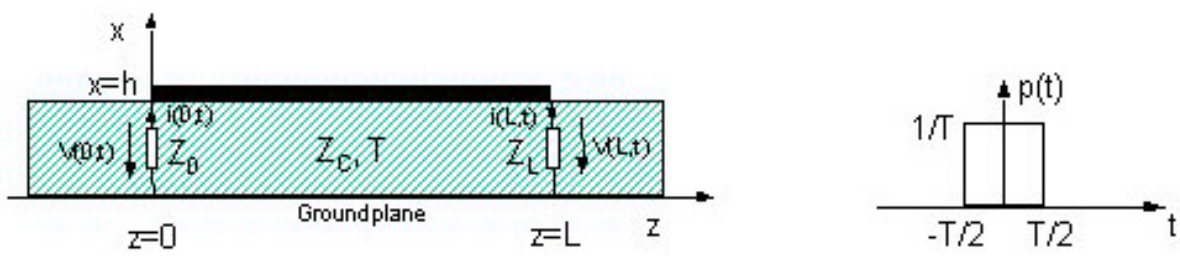

Fig. 2. Transmission line geometry for the model „Single-line above groundplane“.
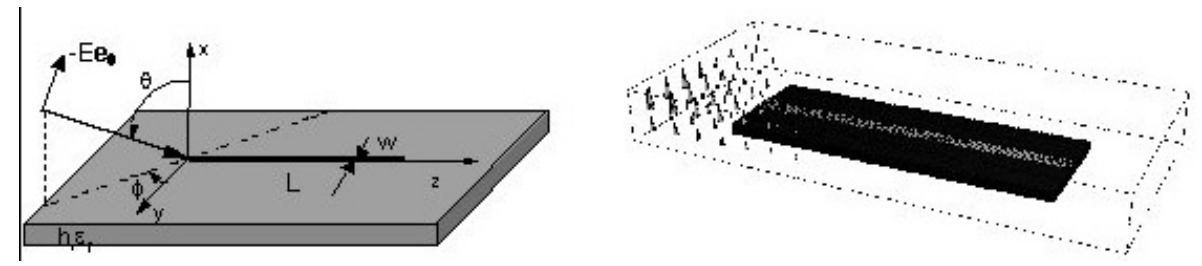

Fig. 3. Transmission line geometry for plane wave excitation.
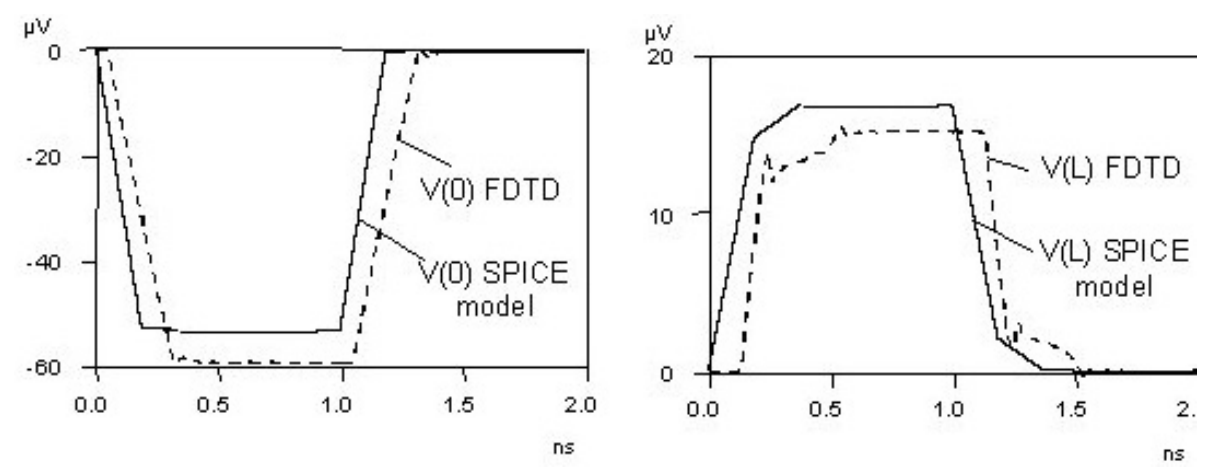

Fig. 4. Case 1: Induced termination voltage $V(0, t)$ and $V(L, t)$ as response to an EM-plane wave excitation by using a step function $E_{0}(t)$ of rise time $t_{r}=1 \mathrm{~ns}\left(t_{r} \sim 5 \times T\right)$.
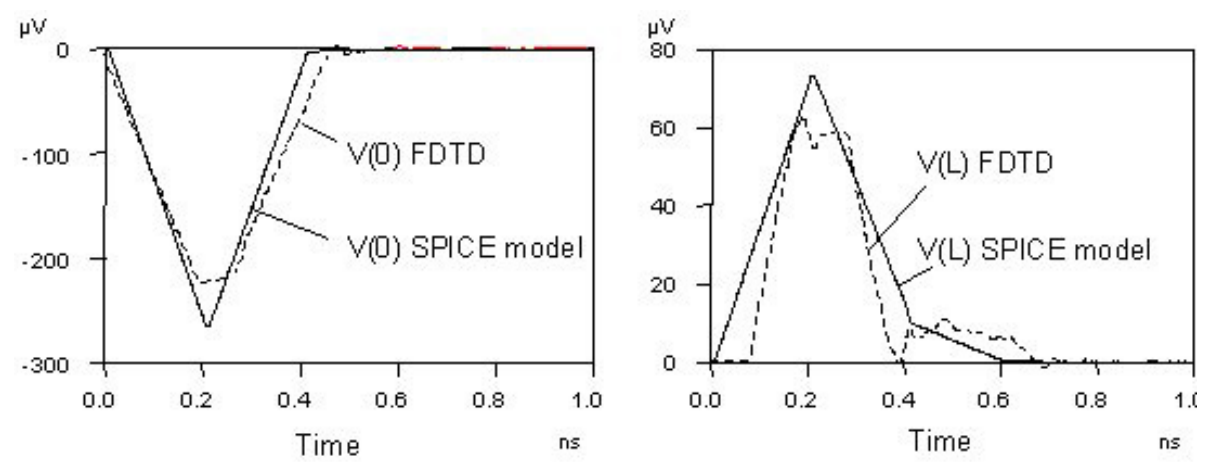

Fig. 5. Case 2: Induced termination voltage $V(0, t)$ and $V(L, t)$ as response to an EM-plane wave excitation by using a step function $E_{0}(t)$ of rise time $t_{r}=0.2 \mathrm{~ns}\left(t_{r} \sim 1 \times T\right)$.

\section{Measurement set-up}

At first the real circuit board will be replaced with a so called sensor board of same size and geometry that is equipped with E- and $\mathrm{H}$-field probes to measure the electric field perpendicular to the PCB groundplane and to measure the magnetic field parallel to the PCB groundplane. It also has connectors to attach all wires leading from and to the circuit board. The measured probe voltages correspond to the driving sources in the simple transmission line model. The transmission line model is completely given in the time domain and implemented in PSPICE with the advantage that any exciting pulse shape and arbitrary load impedances (also non-linear) can be considered. The sensor board is equipped 

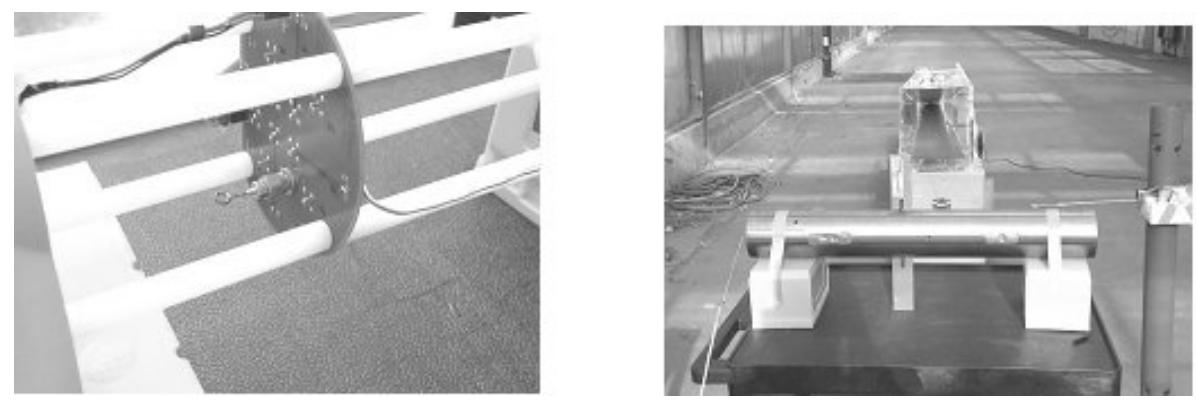

Fig. 6. Sensor board integrated in GENEC body in front of the RADAN-UWB source.

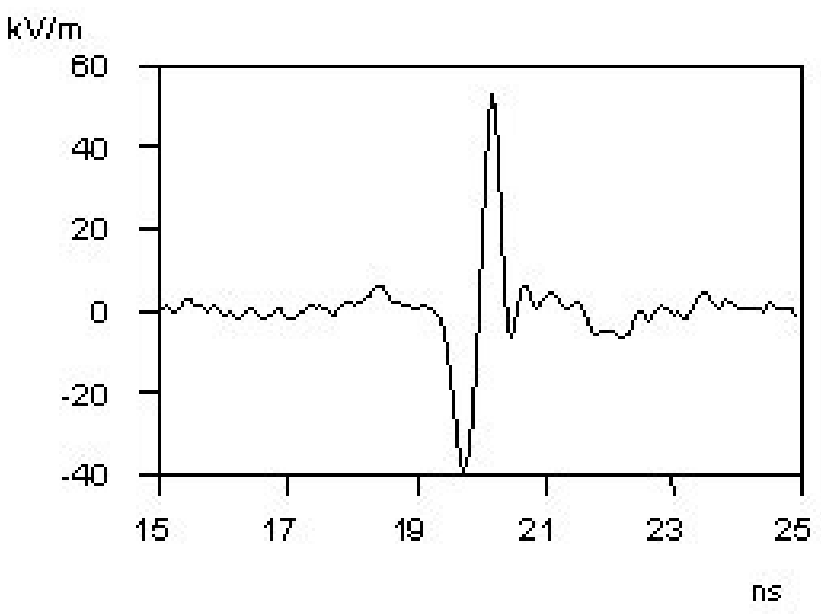

Fig. 7. Incident UWB pulse $E_{\text {inc }}(t)$.

with four Edot monopole probes, one Hdot loop antenna and four distributed $4 \mathrm{~cm}$ long microstrip lines (characteristic line impedance $Z_{C}=50 \mathrm{Ohm}$ ) on a FR4 dielectric of $1 \mathrm{~mm}$ thickness. A $50 \mathrm{Ohm}$ termination load $Z_{L}$ on each line and at both sides is used in this configuration, although other loads are possible (Fig. 1).

The transmission line model is related to Paul (1994) and strongly valid only for a lossless single line over groundplane with $L \ll \lambda$ (Fig. 2). However, good results are still achievable, when the rise time of the incident field $t_{r}$ is of the order of the one way transmission line delay time $T$.

The following SPICE model is used to calculate the induced voltage $V(L, t)$ and $V(0, t)$ at the termination loads $Z_{L}$ and $Z_{0}$ of a microstrip line with the line parameters length $L$, thickness of dielectric $h$ and the characteristic line impedance $Z_{C}$ :

$$
\begin{aligned}
& v(L, t)+Z_{C} i(L, t)=\left[v(0, t-T)+Z_{C} i(0, t-T)\right] \\
& +\left[v_{F}(t-T / 2)+Z_{C} i_{F}(t-T / 2)\right] \times p(t) \\
& v(0, t)-Z_{C} i(0, t)=\left[v(L, t-T)-Z_{C} i(L, t-T)\right] \\
& -\left[v_{F}(t-T / 2)-Z_{C} i_{F}(t-T / 2)\right] \times p(t)
\end{aligned}
$$

with the convolution function $p(t)=\operatorname{rect}(t / T)$ and the oneway transmission line delay $T=L / v$ with $v=c_{0} /\left(\varepsilon_{r, \text { eff }}\right)^{0.5}$.
The driving source voltages $v_{F}$ und $Z_{C} \times i_{F}$ are proportional to the derivatives of the electric field $E_{\perp}$ perpendicular to the groundplane and the magnetic field $B$ parallel to the groundplane and perpendicular to the line axis:

$$
\begin{aligned}
& v_{F}(t) \approx \frac{A}{K_{B}} v_{B}(t) ; v_{B}(t)=K_{B} \cdot \frac{d B}{d t} \\
& i_{F}(t) \approx-\frac{A}{v Z_{C} K_{E} \varepsilon_{r}} v_{E}(t) ; v_{E}(t)=K_{E} \cdot \frac{d E_{\perp}}{d t} .
\end{aligned}
$$

The voltage signals $v_{B}(t)$ and $v_{E}(t)$ are the measured probe voltages, $K_{E}$ und $K_{B}$ the corresponding antenna factors and $A$ is the cross section area $L \cdot h$. For frequencies smaller than $2.5 \mathrm{GHz}$, the probe voltages are proportional to the derivatives of the measured fields.

For model verification purpose, the transmission line is exposed to a plane wave excitation and the results will be compared with a FDTD model (Fig. 3).

The following line parameters are used: $\mathrm{L}=3 \mathrm{~cm}, \mathrm{~h}=1 \mathrm{~mm}$, $\mathrm{w}=1.5 \mathrm{~mm}, \varepsilon_{r}=4.7, Z_{C}=54 \mathrm{Ohm}, Z_{L}=Z_{0}=50 \mathrm{Ohm}$. The electric field $\boldsymbol{E}$ is given as $\boldsymbol{E}(t)=-E_{0}(t) \boldsymbol{e}_{\theta}$ with the angle of incidences $\phi=45^{\circ}$ and $\theta=90^{\circ}$. The time function $E_{0}(t)$ is a step function with rise time $t_{r}$ and amplitude $1 \mathrm{~V} / \mathrm{m}$. Figures 4 and 5 show the comparison between the proposed transmission line SPICE model and the FDTD model for the case $t_{r}=1 \mathrm{~ns}\left(t_{r} \sim 5 \times T\right)$ and for the case $t_{r}=200 \mathrm{ps}\left(t_{r} \sim T\right)$. In both cases good agreement can be noticed.

After the proposed PCB transmission line coupling model was verified with FDTD for uniform plane wave excitation, the sensor PCB was placed in a non-uniform field environment by integrating it in the generic missile system GENEC. The results of the model were compared with a direct line voltage measurement at the PCB. Figure 6 shows the sensor board integrated in the GENEC frame and GENEC body. The coupling paths into the GENEC system are mainly via the attached wings and wing slots. The whole system was excited with an UWB-pulse delivered from a RADAN pulser with attached TEM horn antenna. The incident electric field is vertically polarised parallel to the wing axis at broadside excitation. Figure 7 shows the electric fieldstrength of the incident pulse measured with a D-Dot probe. All signals are recorded with a Tektronix oscilloscope of $4 \mathrm{GHz}$ analog bandwidth. 

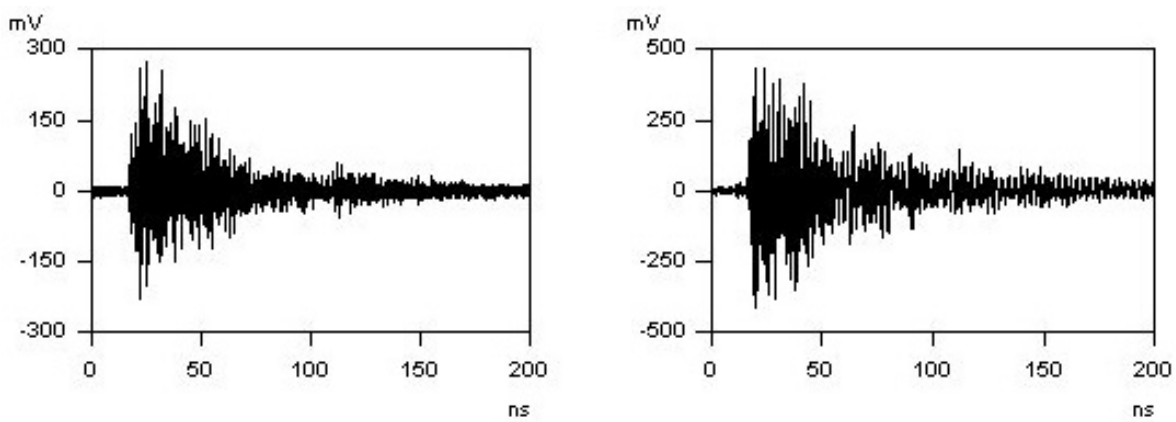

Fig. 8. MP4 - monopole sensor voltage response $v_{E}(t)$ and H-dot loop voltage $v_{B y}(t)$.
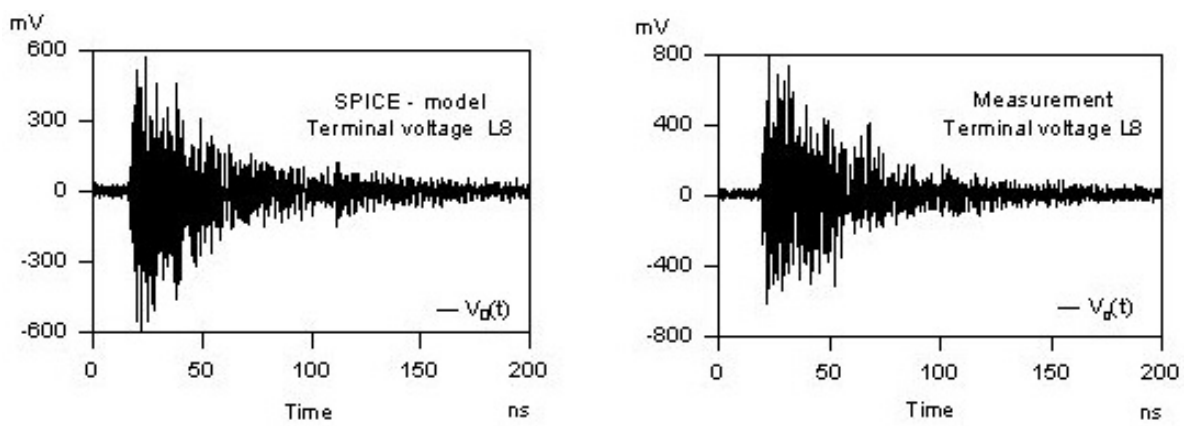

Fig. 9. Terminal voltage $V_{0}(t)$ of line L8 calculated with the SPICE model at $50 \mathrm{Ohm}$ load and measured terminal voltage $V 0(t)$ of line L8 at $50 \mathrm{Ohm}$ load.

According to the coordinate system given in Fig. 1, the electric field of the monopole MP4 and the magnetic field of the loop sensor in y-direction was recorded by using an external trigger signal. Figure 8 shows the measured probe voltages $v_{E}(t)$ and $v_{B y}(t)$ as response to the incident UWBpulse (Fig. 7).

Now, we calculate the induced voltage at the 50 Ohm terminal of line L8 on the sensor PCB (Fig. 1) by using the proposed SPICE model. Because line L8 is orientated in $\mathrm{z}$-direction, we use the magnetic field in the y-direction to calculate the forcing functions $v_{F}(t)$ and $Z_{c} \cdot i_{F}(t)$ with the same line parameters as given in the example before. After convolution with the rect-function $p(t)$, we can implement the forcing functions as voltage controlled voltage sources in the SPICE model.

Figure 9 left shows the result of the L8-line terminal voltage $V_{0}(t)$ calculated with the SPICE model. For comparison, the result of the direct line measurement L8 is depicted on the right with good agreement to the SPICE model.

\section{Conclusions}

The proposed PCB coupling model for a single line over groundplane uses both, a) measurement data of the electric and magnetic field above the board to determine the forcing functions and b) an approximate time-domain SPICE model for electrically short lines similar to (Paul, 1994). Although, we can not expect exact results, the examples show a good approximation achieved with minor effort. This is in many cases enough to estimate the coupled voltages on equivalent boards with similar dimensions and geometry. Furthermore, all the advantages of the time domain SPICE model can be used to treat nonlinear load impedances.

\section{References}

Paul, C. R.: "Analysis of Multiconductor Transmission Lines", John Wiley \& Sons, New York, 1994.

Leone, M. and Singer, H. L.: "On the Coupling of an External Electromagnetic Field to a Printed Circuit Board Trace", IEEE Transaction on Electromagnetic Compatibility, 41, 4, November, 1999.

Erdin, I., Dounavis, A., Achar, R., and Nakhla, M. S.: "A SPICE Model for Incident Field Coupling to Lossy Multiconductor Transmission Lines", IEEE Transaction on Electromagnetic Compatibility, 43, 4, November, 2001.

Tesche, F.: "EMC Analysis Methods and Computational Models", John Wiley and Sons, 1997. 